\section{Importation of Acinetobacter baumannii Into a Burn Unit: A Recurrent Outbreak of Infection Associated With Widespread Environmental Contamination}

\author{
Giorgio Zanetti, MD, MS; Dominique S. Blanc, PhD; \\ Isabelle Federli, RN, CIC; Wassim Raffoul, MD; \\ Christiane Petignat, MD; Philippe Maravic, RN; \\ Patrick Francioli, MD; Mette M. Berger, MD, PhD
}

\begin{abstract}
A burn patient was infected with Acinetobacter baumannii on transfer to the hospital after a terrorist attack. Two patients experienced cross-infection. Environmental swab samples were negative for $A$. baumannii. Six months later, the bacteria reemerged in 6 patients. Environmental swab samples obtained at this time were inoculated into a minimal mineral broth, and culture results showed widespread contamination. No case of infection occurred after closure of the unit for disinfection.
\end{abstract}

Infect Control Hosp Epidemiol 2007; 28:723-725

Acinetobacter baumannii recently gained interest as a cause of dirty wound infection, particularly in mass casualties. ${ }^{1-4}$ It is also a hospital-acquired pathogen that is predominantly found in the intensive care setting. ${ }^{5,6}$ Its propensity to persistently contaminate the hospital environment ${ }^{7}$ and to become resistant to most available antibiotics is a major concern. ${ }^{8,9}$ We describe an outbreak of infection related to an index patient who was transferred to a burn intensive care unit (BICU). This outbreak was characterized by 2 distinct phases in its evolution and by widespread environmental contamination.

\section{METHODS}

The BICU had 3 isolation rooms for burned patients and 1 bay of 4 beds for nonburned surgical intensive care unit patients. No A. baumannii had been cultured from BICU patients during the 4 years that preceded the outbreak. From October 2002 to December 2004, the patients involved in the outbreak were identified from the results of microbiology specimens obtained for clinical management. Screening cultures were not formally performed because many specimens were regularly available, independent of any formal screening culture protocol.

To assess environmental contamination during the first phase of the outbreak, we used swab samples inoculated into brain-heart infusion enrichment broth and subcultured onto MacConkey agar. Suspected A. baumannii colonies were further identified and their susceptibility patterns were deter- mined with microbial identification and susceptibility testing systems (Vitek 2; bioMérieux). During the second phase of the outbreak, swab samples were cultured on minimal mineral broth with acetate, a medium selective for Acinetobacter species. ${ }^{10}$ Because the epidemic strain was resistant to ciprofloxacin, this medium was supplemented with $32 \mathrm{mg} / \mathrm{L}$ of ciprofloxacin (Ciproxin; Bayer AG) to further increase its specificity. All $A$. baumannii isolates recovered during the outbreak, from either patients or the hospital environment, were typed by pulsed-field gel electrophoresis (PFGE) as described elsewhere. ${ }^{11}$

\section{RESULTS}

First phase. The index patient, whose burns resulted from the October 12, 2002, bombing in Bali, was transferred to the BICU 4 days later and isolated under contact precautions (Figure 1). On the day of transfer, A. baumannii was cultured from wound biopsy specimens, from the tips of 3 intravascular catheters, and from sputum specimens. The isolate was fully susceptible only to carbapenems and amikacin.

Two of 3 consecutive burned patients became infected with a strain of A. baumannii that had the same PFGE profile. This was not the case for 113 nonburned patients who stayed in the BICU during the same period.

We collected environmental swab samples from the hydrotherapy room, because it was shared by burned patients only, but found no A. baumannii. Transmission precautions were reinforced. A single cohort of nurses took care of all $A$. baumannii-positive patients. No additional case of infection occurred among the 14 burned patients admitted to the hospital during the 6 following months.

Second phase. After this interruption, 6 of 9 consecutive burned patients were infected with $A$. baumannii, but again none of the 91 nonburned patients was infected. We obtained 161 swab samples from the environment, which this time were cultured on minimal mineral broth with acetate supplemented with ciprofloxacin. A. baumannii was recovered from $16(10 \%)$ of 161 swab samples, mostly from the hydrotherapy room and a patient's room. The environmental strains and the patients' strains had a PFGE profile that was indistinguishable from that of the isolate recovered during the first phase of the outbreak (Figure 2).

We implemented more stringent and extensive cleaning procedures, including a ban against stockpiling any material in the rooms and a requirement to replace unused, exposed material after each procedure in the hydrotherapy room and the operating room, and daily in the patients' rooms. Finally, the BICU was closed to new patients until the last A. baumannii-positive patient could be discharged to the regular ward (ie, for 2.5 months). As a consequence, admission had to be denied to 9 burned patients who were transferred to 


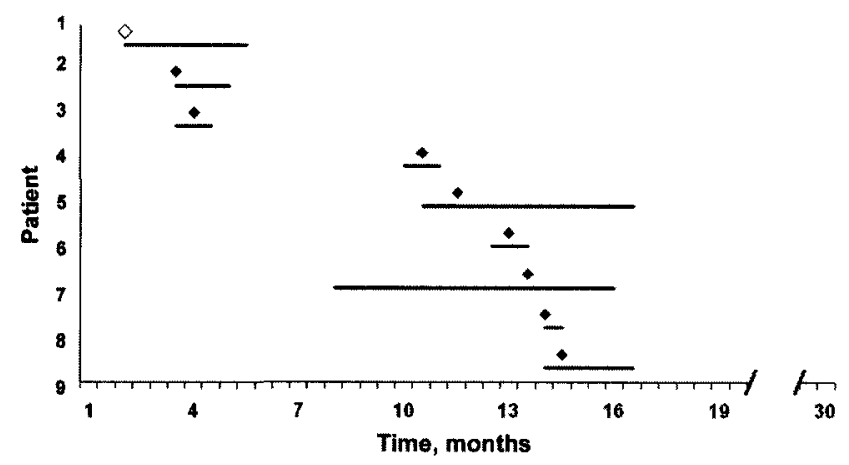

FIGURE 1. Time distribution of Acinetobacter baumannii infection in the 9 burn unit patients in the outbreak. Bar, length of stay in the unit for 1 patient involved in the outbreak. Diamonds, first occurrence of A. baumannii-positive culture results (open diamond, index case; closed diamonds, secondary cases).

another burn center. Before reopening, the BICU underwent an extensive chemical-mechanical disinfection, and material and equipment were sterilized, disinfected, and/or replaced. Control swab samples collected from environmental surfaces were negative for $A$. baumannii at that time and again 8 months thereafter. No new case of infection due to $A$. baumannii occurred among the 32 burn patients admitted during the 18-month follow-up period after the BICU reopened.

\section{DISCUSSION}

This case series illustrates that hospitals are vulnerable to importation of A. baumannii when patients are transferred from other hospitals, ${ }^{12}$ from a battlefield, or from the scene of a mass catastrophe. ${ }^{2-4}$ Similar to the index patient, several survivors of the terrorist attack in Bali were colonized with A. baumannii. ${ }^{1}$

The patients who were infected with $A$. baumannii in this outbreak were hospitalized for significantly longer periods because of delayed wound healing. Three patients experienced septic shock-related bowel perforation, which was fatal for one of them (data not shown).

Most outbreaks caused by $A$. baumannii have involved intensive care unit patients, ${ }^{7}$ particularly burn patients. ${ }^{13-15}$ In our series, A. baumannii not only did not cause infection outside the BICU, but it was never recovered from nonburned surgical ICU patients treated in the BICU. This observation suggested the hypothesis that the burned patients shared a common exposure. Indeed, debridement surgery and hydrotherapy were examples of invasive procedures that these patients underwent in the same rooms.

We were unable to demonstrate contamination of the environment during the first phase of the outbreak, similar to $26(51 \%)$ of $51 \mathrm{~A}$. baumannii epidemics described in the literature from 1977 through 2000. Environmental contamination may have been absent or minimal during this phase. Alternatively, it is possible that this contamination was not documented because the first-phase investigation was less intensive than that during the second phase or because the brain-heart infusion enrichment broth that was used during the first phase was not sufficiently sensitive to detect $A$. baumannii in the environment. We eventually found a fairly disseminated contamination of the environment when we used minimal mineral broth with acetate ${ }^{10}$ during the second phase. Contamination of material and surfaces was obvious in patients' rooms and in the hydrotherapy room. One may consider patients undergoing hydrotherapy at high risk for environmental contamination, because hydrotherapy is associated with moisture and high temperature in the room, droplet dispersion, and intensive handling of anesthetized patients with large, contaminated wounds, which create numerous opportunities for breakdowns in contact precautions. The receipt of hydrotherapy was indeed an independent risk factor for A. baumannii bloodstream infection in burn patients in a study by Wisplinghoff et al. ${ }^{15}$ The presence of $A$. baumannii on boxes of an emergency drug (norepinephrine) illustrates the importance of complying with isolation precautions even under critical circumstances.

We have no clear explanation of how and where the $A$. baumannii epidemic strain persisted between the 2 phases of the outbreak. We could not find any change in care procedures or in the population of either patients or healthcare workers that could represent reexposure to a persisting source. A persisting environmental source was not looked for during the interval between the 2 phases, because we assumed that the problem was resolved at that time. The known propensity of $A$. baumannii to persistently contaminate surfaces is a likely explanation for the resurgence of the outbreak. ${ }^{16,17}$

Events of this kind illustrate that even hospitals without endemic A. baumannii are at risk of importing this bacterium via transfer of patients. Microbiological screening is advisable for patients transferred from endemic areas, battlefields, or the scenes of mass catastrophes, along with strict infection

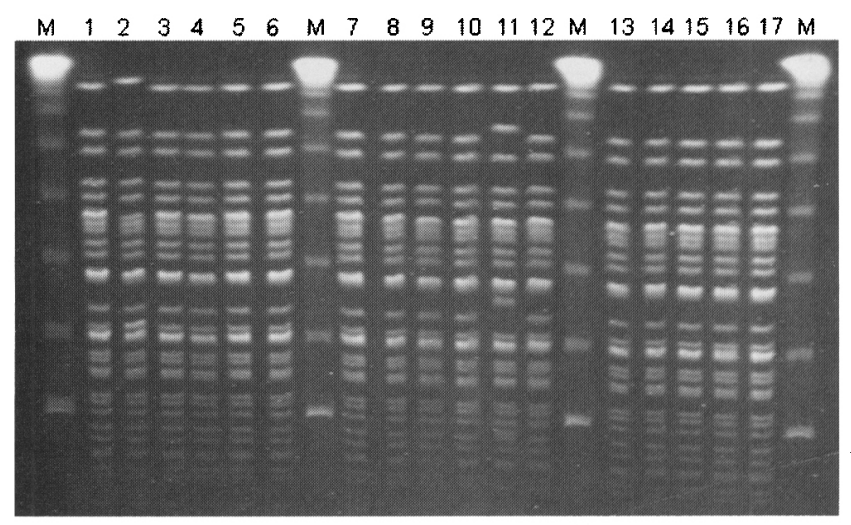

FIGURE 2. Pulsed-field gel electrophoresis patterns after digestion with restriction enzyme ApaI of DNA from Acinetobacter baumannii isolates from 6 of the 9 patients (lanes 1-6) and from the environment (lanes 7-17). M, molecular markers. 
control measures. If secondary cases occur, the detection of environmental contamination by use of a minimal mineral broth with acetate may help guide measures to interrupt transmission.

\section{ACKNOWLEDGMENTS}

Potential conflicts of interest. All authors report no conflicts of interest relevant to this article.

From the Service of Hospital Preventive Medicine (G.Z., D.S.B., I.F., C.P., P.F.), the Service of Infectious Diseases, Department of Medicine (G.Z., P.F.), the Service of Plastic and Reconstructive Surgery, Department of Surgery (W.R.), and the Service of Surgical Intensive Care and Burn Center, Department of Surgery (P.M., M.M.B.), University Hospital, Lausanne, Switzerland.

Address reprint requests to Giorgio Zanetti, MD, MS, Service of Hospital Preventive Medicine, Centre Hospitalier Universitaire Vaudois, 1011 Lausanne, Switzerland (Giorgio.Zanetti@chuv.ch).

Received June 22, 2006; accepted September 25, 2006; electronically published May 11, 2007.

(C) 2007 by The Society for Healthcare Epidemiology of America. All rights reserved. 0899-823X/2007/2806-0015\$15.00. DOI: $10.1086 / 517956$

\section{REFERENCES}

1. Christiansen KJ, MacCullough C, Coombs G. Multiresistant organisms isolated from victims of the Bali terrorist bombing. In: Program and abstracts of the 43rd Interscience Conference on Antimicrobial Agents and Chemotherapy (Chicago). Washington, DC: American Society for Microbiology, 2003:359. Abstract K696.

2. Deye GA, Riddell SW, Bradley W, Endy TP. Microbiology of infected traumatic war wounds in operation Iraqi Freedom. In: Program and abstracts of the 42nd Annual Meeting of the Infectious Diseases Society of America (Boston). Alexandria, VA: Infectious Diseases Society of America, 2004:256. Abstract LB-9.

3. Herper M. The Iraq infection. Promed; 3-8-2005. Available at: http://www promedmail.org. Accessed June 20, 2006. Archive no. 20050803.2248.

4. Scott PT, Petersen K, Fishbain J. Acinetobacter baumannii infections among patients at military medical facilities treating injured USA service members, 2002-2004. Promed; 19-11-2004. Available at: http://www .promedmail.org. Accessed June 20, 2006. Archive no. 20041119.3105.

5. Villegas MV, Hartstein AI. Acinetobacter outbreaks, 1977-2000. Infect Control Hosp Epidemiol 2003; 24:284-295.

6. Wisplinghoff H, Edmond MB, Pfaller MA, Jones RN, Wenzel RP, Seifert $\mathrm{H}$. Nosocomial bloodstream infections caused by Acinetobacter species in United States hospitals: clinical features, molecular epidemiology, and antimicrobial susceptibility. Clin Infect Dis 2000; 31:690-697.

7. Urban C, Segal-Maurer S, Rahal JJ. Considerations in control and treatment of nosocomial infections due to multidrug-resistant Acinetobacter baumannii. Clin Infect Dis 2003; 36:1268-1274.

8. Van Looveren M, Goossens H. Antimicrobial resistance of Acinetobacter spp. in Europe. Clin Microbiol Infect 2004; 10:684-704.

9. Rodriguez-Bano J, Cisneros JM, Fernandez-Cuenca F, et al. Clinical features and epidemiology of Acinetobacter baumannii colonization and infection in Spanish hospitals. Infect Control Hosp Epidemiol 2004; 25: 819-824.

10. Dijkshoorn L, Van Vianen W, Degener JE, Michel MF. Typing of Acinetobacter calcoaceticus strains isolated from hospital patients by cell envelope protein profiles. Epidemiol Infect 1987; 99:659-667.

11. van Dessel H, Dijkshoorn L, van der Reijden T, et al. Identification of a new geographically widespread multiresistant Acinetobacter baumannii clone from European hospitals. Res Microbiol 2004; 155:105-112.

12. Kaiser AM, Schultsz C, Kruithof GJ, bets-Ossenkopp Y, VandenbrouckeGrauls $\mathrm{C}$. Carriage of resistant microorganisms in repatriates from foreign hospitals to The Netherlands. Clin Microbiol Infect 2004; 10:972-979.

13. Ferreira AC, Gobara S, Costa SE, et al. Emergence of resistance in Pseudomonas aeruginosa and Acinetobacter species after the use of antimicrobials for burned patients. Infect Control Hosp Epidemiol 2004; 25: 868-872.

14. Wong TH, Tan BH, Ling ML, Song C. Multi-resistant Acinetobacter baumannii on a burns unit: clinical risk factors and prognosis. Burns 2002; 28:349-357.

15. Wisplinghoff $H$, Perbix W, Seifert $H$. Risk factors for nosocomial bloodstream infections due to Acinetobacter baumannii: a case-control study of adult burn patients. Clin Infect Dis 1999; 28:59-66.

16. Wendt C, Dietze B, Dietz E, Ruden H. Survival of Acinetobacter baumannii on dry surfaces. J Clin Microbiol 1997; 35:1394-1397.

17. Bernards AT, Harinck HI, Dijkshoorn L, van der Reijden TJ, van den Broek PJ. Persistent Acinetobacter baumannii? look inside your medical equipment. Infect Control Hosp Epidemiol 2004; 25:1002-1004. 